\title{
INDUCTIVE LEARNING OF COMPLEX FUZZY RELATION
}

\author{
S. K. Das ${ }^{1}$, D.C. Panda ${ }^{2}$, Nilambar Sethi ${ }^{3}$ and S. S. Gantayat ${ }^{3}$ \\ ${ }^{1}$ Department of Computer Science, Berhampur University, Berhampur, Orissa \\ dr.dassusanta@yahoo.co.in \\ ${ }^{2}$ Department of Electronics \& Communication Engineering \\ Jagannath Institute of Technology \& Management, Paralakhemundi, Orissa \\ d_c_panda@yahoo.com \\ ${ }^{3}$ Department of Information Technology \\ Gandhi Institute of Engineering \& Technology, Gunupur, Orissa \\ nilambar_sethi@rediffmail.com \\ sgantayat67erediffmail.com
}

\begin{abstract}
The objective of this paper to investigate the notion of complex fuzzy set in general view. In constraint to a traditional fuzzy set, the membership function of the complex fuzzy set, the range from [0.1] extended to a unit circle in the complex plane. In this article the comprehensive mathematical operations on the complex fuzzy set are presented. The basic operation of complex fuzzy set such as union, intersection, complement of complex fuzzy set and complex fuzzy relation are studied. Also vector aggregation and fuzzy relation over the complex fuzzy set are discussed. Two novel operations of complement and projection for complex fuzzy relation are introduced.
\end{abstract}

\section{KEY WORD}

Complex fuzzy set, complex fuzzy relation, complement of complex fuzzy relation, projection of complex fuzzy relation.

\section{INTRODUCTION}

The notion of the fuzzy set was first introduced by Zadeh in a seminal paper in 1965 [7]. The notion of the fuzzy set $A$ on the universe of discourse $U$ is the set of order pair $\left\{\left(x, \mu_{A}(x)\right), x \varepsilon U\right\}$ with a membership function $\mu_{\mathrm{A}}(\mathrm{x})$, taking the value on the interval [0,1]. Ramot et al [5], extended the fuzzy set to complex fuzzy set with membership function, $z=r_{s} e^{i w_{s}(x)}$ where $i=\sqrt{-1}$, which ranges in the interval [0,1] to a unit circle. Ramot et al [4], also introduced different fuzzy complex operations and relations, like union, intersection, complement etc., Still it is necessary to determine the membership functions correctly, which will give the appropriate or approximate result for real life applications. The membership function defined for the complex fuzzy set $z=r_{s} e^{i w_{s}(x)}$, which compromise an amplitude term $\mathrm{r}_{\mathrm{s}}(\mathrm{x})$ and phase term $\mathrm{w}_{\mathrm{s} .}$ The amplitude term retains the idea of "fuzziness" and phase term signifies declaration of complex 
fuzzy set, for which the second dimension of membership is required. The complex fuzzy set allows extension of fuzzy logic that is i to continue with one dimension gradeness of membership. Xin Fu et al [8] defined the fuzzy complex membership function of the form $z=a+i b$, where $x, y$ are two fuzzy numbers with membership function $\mu_{\mathrm{A}}(\mathrm{x}), \mu_{\mathrm{B}}(\mathrm{x})$ respectively. If $\mathrm{b}$ does not exist, $\mathrm{z}$ degenerates to a fuzzy number.

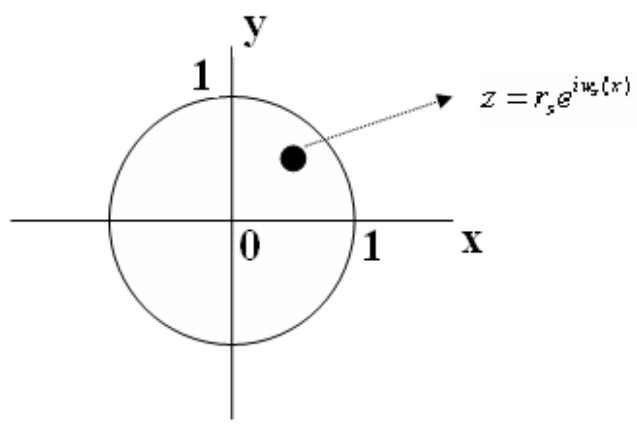

Figure 1.1. Fuzzy Complex Number

$\mathrm{Xin} \mathrm{Fu}$ et al [8], also discussed a complex number in cartesian form where $\mathrm{a}=\mathrm{r}_{\mathrm{s}} \cos (\mathrm{x})$ and $\mathrm{b}=\mathrm{r}_{\mathrm{s}} \sin (\mathrm{x})$, which are in polar forms defined in [4]. The fuzzy number is created by interpolating complex number in the support of fuzzy set ([1], [2], [3][6]).

Complex fuzzy set is a unique framework over the advantage of traditional fuzzy set .The support of complex fuzzy set is unrestricted, may include any kind of object such as number, name etc, which is off course a complex number. The notion of T-norm and T-conorm are used through out this paper [4].

\subsection{Definition T-norm (Jang et al [4]).}

Four of the most frequently used T-norm operators are

$$
\begin{array}{ll}
\text { Minimum } & \mathrm{T}_{\min }(\mathrm{a}, \mathrm{b})=\min (\mathrm{a}, \mathrm{b})=\mathrm{a} \wedge \mathrm{b} \\
\text { Algebraic Product } & \mathrm{T}_{\mathrm{ap}}(\mathrm{a}, \mathrm{b})=\mathrm{ab} . \\
\text { Bounded product } & \mathrm{T}_{\mathrm{bp}}(\mathrm{a}, \mathrm{b})=0 \vee(\mathrm{a}+\mathrm{b}-1) \\
\text { Drastic product } & \mathrm{T}_{\mathrm{dp}}(\mathrm{a}, \mathrm{b})= \begin{cases}\mathrm{a}, & \text { if } \mathrm{b}=1 \\
\mathrm{~b}, & \text { if } \mathrm{a}=1 \\
0, & \text { if } \mathrm{a}, \mathrm{b}<1\end{cases}
\end{array}
$$

With understanding that $\mathrm{a}$ and $\mathrm{b}$ are between 0 and 1 .

\subsection{Definition T-Conorm (Jang et al [4])}

Corresponding to four T-norm operators in the previous definition we have the following four T-conorm operators.
Maximum
Algebraic Sum
$\mathrm{S}_{\max }(\mathrm{a}, \mathrm{b})=\max (\mathrm{a}, \mathrm{b})=\mathrm{a} \vee \mathrm{b}$
Bounded product
$S(a, b)=a+b-a b$
$S(a, b)=1 \wedge(a+b)$ 
Drastic product $S(a, b)= \begin{cases}a, & \text { if } b=0 \\ b, & \text { if } a=0 \\ 1, & \text { if } a, b>1\end{cases}$

This paper is organized in the following order. The review of complex fuzzy set and operations on it are discussed in section 2 and section 3 the complex fuzzy relation and operations are mentioned on them. A novel idea to vector aggregation, complement of complex fuzzy relation and projection of complex fuzzy relation are studied in section 4 followed by summary and suggestion for future work are given in section 5 .

\section{COMPlex Fuzzy SeT}

\subsection{Concept \& Operations}

Complex fuzzy set is the basis for complex fuzzy logic [7]. The formal definition of complex fuzzy set was provided in [7] followed by a discussion and interpretation of the moral concept. In addition, several set theoretic operations on complex fuzzy set were discussed with several examples, which illustrate the potential applications of complex fuzzy set in information processing.

\subsection{Definition of Complex Fuzzy Set}

A complex fuzzy set $\mathrm{S}$, defined on a universe of discourse $\mathrm{U}$, is characterized by a membership function $\mu_{s}(x)$, which can assign any element $\mathrm{x} \in \mathrm{U}$ as a complex valued grade of membership in S. The value of $\mu_{s}(x)$ lies in a unit circle in the complex plane and in the form $z=r_{s} e^{i w_{s}(x)}$, where $i=\sqrt{-1}$ and $\mathrm{r}_{\mathrm{s}} \in[0,1]$.

The complex fuzzy set S, may be defined as the set of order pair given by

$$
S=\left\{\left(x, \mu_{s}(x)\right): x \in U\right\}
$$

\subsection{Complex Fuzzy Union}

\subsubsection{Definition $([6,7])$.}

In the traditional fuzzy logic, union for two fuzzy set $A$ and $B$ on $U$ denoted as $A \cup B$ is specified by a function $\mu$, where $\mu:[0,1] \times[0,1] \rightarrow[0,1]$.

The membership function $\mu_{(\mathrm{A} \cup \mathrm{B})}$, may be defined as one of followings.

$$
\begin{array}{ll}
\text { (i) Standard Union } & \mu_{A U B}(x)=\max \left[\mu_{A}(x), \mu_{B}(x)\right] \\
\text { (ii) Algebraic Sum } & \mu_{A U B}(x)=\mu_{A}(x)+\mu_{B}(x)-\mu_{A}(x) \cdot \mu_{B}(x) \\
\text { (iii) Bound Sum } & \mu_{A U B}(x)=\min \left[1, \mu_{A}(x)+\mu_{B}(x)\right]
\end{array}
$$


International Journal of Computer Science, Engineering and Information Technology (IJCSEIT), Vol.1, No.5, December 2011

The fuzzy union must satisfy the definition 2.2 and is equivalent to the proposed T-Co norms [9].

\subsubsection{Properties}

The Complex fuzzy union is defined by a set of axioms. These axioms represent properties of complex fuzzy union functions that must satisfy in order to intuitively acceptable values.

(a) Complex fuzzy union does not satisfy the closure property.

In the algebraic sum union function does not satisfy the closure property for complex fuzzy set, which can be realized from the following example.

Example 2.3.1. $\mu_{A}(x)=\mu_{B}(x)=i$

$$
\Rightarrow \mu_{A \cup B}{ }^{(x)}=\mu_{A}{ }^{(x)}+\mu_{B}{ }^{(x)}-\mu_{A}{ }^{(x)} \cdot \mu_{B}{ }^{(x)}=1+2 i
$$

It shows that the membership function lies outside of the unit circle. i.e. $\sqrt{1^{2}+2^{2}}=\sqrt{5}>1$.

(b) Complex Fuzzy union must be monotonic

Since complex number is not linearly order, monotonicity of the complex numbers is required.

Example 2.3.2. Suppose $b \leq d \Rightarrow \mu(a, b) \leq \mu(a, d)$ using definition2.3.1 (2)

Similarly the max and min operators used in (3) \& (5) are not applicable to complex valued grades of membership. Here we can apply traditional fuzzy definition of union by keeping same approach to complex part and different approach to phase may be defined in the following way.

\subsubsection{Definition $([6,7])$.}

Suppose A and B be two complex fuzzy set on $U$ with complex valued membership function $\mu_{n}(x), \mu_{B}(x)$ respectively. Then complex fuzzy union $\mathrm{A} \cup \mathrm{B}$ may be defined as

$$
\mu_{(A \cup B)}(x)=\left[r_{A}(x) \oplus r_{B}(x)\right] e^{i w_{A \cup B}(x)}
$$

where $\oplus$ represents the T-Conorm and $W_{A \cup B}$ is defined as follows.
a) (Sum) $W_{A \cup B}=W_{A}+W_{B}$
b) $\quad(\operatorname{Max}) W_{A \cup B}=\max \left(W_{A}, W_{B}\right)$
c) (Min) $W_{A \cup B}=\min \left(W_{A}-W_{B}\right)$

and

d) (Winner takes all) $W_{A U B}=\left\{\begin{array}{lll}W_{A} & \text { if } r_{A}>r_{B} \\ W_{B} & \text { if } r_{B} \geq r_{A}\end{array}\right.$

To illustrate the above, consider the following example. 
Example 2.3.3: Suppose $A=\left\{\frac{1 \mathrm{e}^{\mathrm{i} \times 0}}{-1}, \frac{0.4 \mathrm{e}^{\mathrm{i} \pi}}{0}, \frac{0.8 \mathrm{e}^{\mathrm{i} \pi / 2}}{1}\right\}$ and $B=\left\{\frac{0.2 \mathrm{e}^{\mathrm{i} 3 \pi / 4}}{-1}, \frac{0.3 \mathrm{e}^{\mathrm{i} 2 \pi}}{0}, \frac{1 \mathrm{e}^{\mathrm{i} \pi / 5}}{1}\right\}$

Then $A \cup B=\left\{\frac{1 \mathrm{e}^{0 \pi}}{-1}, \frac{0.4 \mathrm{e}^{\mathrm{i} \pi}}{0}, \frac{1 \mathrm{e}^{\mathrm{i} \pi / 5}}{1}\right\}$

Here the union operation is (1) of definition 2.3.1.

\subsection{Fuzzy Complex Intersection}

It should be noted that the derivation of fuzzy complex intersection closely related to complex fuzzy union, which clear from the following definition.

2.4.1. Definition([6,7]). The intersection for two complex fuzzy set A and $\mathrm{B}$ on $\mathrm{U}$ defined as

$$
A \cap B=\left[r_{A}(x) * r_{B}(x)\right] e^{i w_{A \cap B}(x)}
$$

where $*$ is denoted as $\mathrm{T}$-norm.

Example 2.4.1. From the example 2.3.3, we can find the fuzzy intersection of the two complex fuzzy sets A and B as follows.

$$
A \cap B=\left\{\frac{0.2 \mathrm{e}^{\mathrm{i} 3 \pi / 4}}{-1}, \frac{0.3 \mathrm{e}^{\mathrm{i} 2 \pi}}{0}, \frac{0.8 \mathrm{e}^{\mathrm{i} 5 \pi / 2}}{1}\right\}
$$

Note: The fuzzy union and intersection are not unique, As this depend on the type of functions used in T-Conorm and T-norms.

\subsection{Complex Fuzzy Complement}

We known that the complement of a fuzzy set $\mathrm{A}$ on $\mathrm{U}$ with membership function $\mu_{A}(x)$ is defined as, $\left(1-\mu_{A}(x)\right.$.)

The similar concept can be applied to the complex fuzzy set. But it is not always true for some cases. To illustrate this, consider the following example.

Example 2.5.1. Suppose the membership function of a complex fuzzy set is $\mu_{s}(x)=1 e^{i \pi}$.

That is, $\mu_{s}(x)=1 e^{i \pi}=1[\cos \pi+i \sin \pi]=-1$

So the complement of $\mu_{s}(x)=1 e^{i \pi}$ is $\mu_{s}(x)=1-(-1)=2$, which is outside of the unit circle. This is violating of the closure properties of Fuzzy complex number.

2.5.1. Definition ([6,7]). Suppose A is a fuzzy set defined as $U$. The complex fuzzy complement for the fuzzy set $\mathrm{A}$ is defined as $\left(1-\mu_{A}(x)\right)$, where $\mu_{A}(x)=r_{s}(x)\left[\cos w_{s}(x)+\sin w_{s}(x)\right]$

This form of complex fuzzy complement does not reduce to its traditional fuzzy logic, if $w_{s}(x)=0$. That is, 
International Journal of Computer Science, Engineering and Information Technology (IJCSEIT), Vol.1, No.5, December 2011

when $\mu_{s}(x)=0, C\left(\mu_{s}(x)\right)=\left[1-r_{s}(x)\right]+i$ which is again a complex number.

Hence we can write $C\left(\mu_{s}(x)\right)=C\left(r_{s}(x)\right) \cdot e^{i c\left(w_{s} x\right)}$

Consider the function $\mathrm{C}:[0,1] \rightarrow[0,1]$, if $\mu_{s}(x)=C\left(\mu_{s}(x)\right)$ then the equation (13) holds. So we need $\left|C \cdot \mu_{s}(x)\right|=C\left(\left|\mu_{s}(x)\right|\right)$ to satisfy the equation (13). This can be achieved by adding $\pi$ to $w_{s}(x)$.

Hence the complement of $w_{s}(x)$ will be $w_{s}(x)+\pi$ that is $C\left(\mu_{s}(x)\right)=C \mu_{s}(x)+\pi$.

\section{COMPLEX Fuzzy RELATION}

In this section, the complex fuzzy relation is introduced. Here discussion is limited to the relation between two complex fuzzy set.

3.1. Definition (Complex Fuzzy Relation) ([10]): Let A and B be two complex fuzzy sets defined on $U$. Then

$$
\mu_{A}(x)=r_{A}(x) \cdot e^{i w_{A}(x)} \text { and } \mu_{B}(x)=r_{B}(x) \cdot e^{i w_{B}(x)}
$$

are their corresponding membership values. We can say $A \leq B$ if and only if both amplitude and phase of $\mathrm{A}$ and $\mathrm{B}$ are $A \leq B$ that is $r_{A}(x) \leq r_{B}(x)$ and $w_{A}(x) \leq w_{B}(x)$.

A complex fuzzy relation $\mathrm{R}(\mathrm{U}, \mathrm{V})$ is a complex fuzzy subset of the product space $\mathrm{U} \times \mathrm{V}$. The relation $\mathrm{R}(\mathrm{U}, \mathrm{V})$ is characterized by a complex membership function

$$
\begin{aligned}
& \mu_{R}(x, y), x \in U, y \in V \\
& \text { Where } R(U, V)=\left\{\left((x, y), \mu_{R}(x, y)\right) \mid \forall(x, y) \in U \times V\right\}
\end{aligned}
$$

Here the membership function $\mu_{R}(x, y)$ has the value within an unit circle in the complex plane with the from $\mu_{R}(x, y)=r(x, y) \cdot e^{i w(x, y)}$

where $e^{i w(x, y)}$ is a periodic function defined as $w(x, y)=w(x, y)+2 k \pi, k=0, \pm 1, \pm 2$

\subsection{Definition ([10])}

Let $\mathrm{X}, \mathrm{Y}$ and $\mathrm{Z}$ be three different Universes. Suppose A be a complex fuzzy relation defined from $\mathrm{X}$ to $\mathrm{Y}$ and $\mathrm{B}$ be a complex fuzzy relation defined from $\mathrm{Y}$ to $\mathrm{Z}$. Then composition of $\mathrm{A}$ and $\mathrm{B}$ denoted on $\mathrm{AoB}$ is a complex fuzzy relation defined from $\mathrm{X}$ to $\mathrm{Z}$ which can be represented as

$$
\mu_{A o B}(x, z)=r_{A o B}(x, z) \cdot e^{i w_{A o B}(x, z)}=\sup _{\forall y} \inf \left(r_{A}(x, y), r_{B}(y, z)\right) \cdot e^{i \sup \left(\inf \left(w_{A}(x, y), w_{B}(y, z)\right)\right.}
$$




\subsection{Definition (Complex Fuzzy Union Relation) ([10])}

Let $\mathrm{A}$ and $\mathrm{B}$ be two complex fuzzy relations defined on $\mathrm{U} x \mathrm{~V}$. The corresponding membership functions of $\mathrm{A}$ and $\mathrm{B}$ are defined as

$\mu_{A}(x, y)=r_{A}(x, y) \cdot e^{i w_{A}(x, y)}$ and $\mu_{B}(x, y)=r_{B}(x, y) \cdot e^{i w_{B}(x, y)}$ respectively.

The complex fuzzy union relation of $\mathrm{A}$ and $\mathrm{B}$ is defined as $\mathrm{A} \cup \mathrm{B}$ by membership function $\mu_{A \cup B}(x, y)$ where

$\mu_{A \cup B}(x, y)=r_{A U B}(x, y) \cdot e^{i w_{A U B}(x, y)}=\max \left(r_{A}(x, y), r_{B}(x, y)\right) \cdot e^{i \max \left(w_{A}(x, y), w_{B}(x, y)\right)}$

where $r_{A \cup B}(x, y)$ is a relation valued function on $[0,1]$ and $e^{i w_{A \cup B}(x, y)}$ is a periodic function defined in (18)

\subsection{Definition (Complex Fuzzy Intersection Relation) ([10])}

Let $\mathrm{A}$ and $\mathrm{B}$ be two complex fuzzy sets defined on $\mathrm{U} x \mathrm{~V}$ with their corresponding membership functions as

$$
\mu_{A}(x, y)=r_{A}(x, y) e^{i w_{A}(x, y)} \text { and } \mu_{B}(x, y)=r_{B}(x, y), e^{i w_{B}(x, y)}
$$

respectively. The complex fuzzy intersection relation of $\mathrm{A}$ and $\mathrm{B}$ is defined as $\mathrm{A} \cap \mathrm{B}$ by membership function $\mu_{A \cap B}(x, y)$

where $\mu_{A \cap B}(x, y)=r_{A \cap B}(x, y) \cdot e^{i w_{A \cap B}(x, y)}$

$$
=\min \left(r_{A}(x, y), r_{B}(x, y)\right) \cdot e^{i \min \left(w_{A}(x, y), w_{B}(x, y)\right)}
$$

Here $r_{A \cap B}(x, y)$ is a relation valued function on $[0,1]$ and $e^{i w_{A \cap B}(x, y)}$ is a periodic function.

\section{VECTOR AGGREGATION FOR COMPLEX FUZZY SET:}

It allows the aggregation of complex fuzzy set which incorporate phase consideration.

\subsection{Definition (Vector Aggregation) ([7])}

Suppose $C_{1}, C_{2} \ldots \ldots C_{n}$ be complex fuzzy sets on $U$. The vector aggregation of $C_{1}, C_{2} \ldots \ldots C_{n}$ may be defined by a function $v$ as

$v:\{a: a \in C,|a| \leq 1\}^{n} \rightarrow\{b: b \in C,|b| \leq 1\}$ for all $x \in U, v$ is given by

$\mu_{A}(x)=v\left(\mu_{A 1}(x), \mu_{A 2}(x) \ldots \ldots \ldots \ldots \ldots . . . \mu_{A n}(x)\right)=\sum_{i=1}^{n} w_{i} \mu_{A i}(x)$

where $w_{i} \in\{a|a \in C| a \mid, \leq 1\}$ for all $i$ and $\sum_{c=1}^{n}\left|w_{i}\right|=1$

Note: In Complex vector aggregation, the amplitude of the sum may reduce to individual amplitude. 
International Journal of Computer Science, Engineering and Information Technology (IJCSEIT), Vol.1, No.5, December 2011

\subsection{Definition (Complement of Complex Fuzzy Relation)}

In traditional fuzzy set, the complement of a relation $\mathrm{R}$ denoted as $\bar{R}$ defined by $\mu_{\bar{R}}(x, y)=1-\mu_{R}(x, y), \forall x, y \in U \times V$

Suppose the complex fuzzy relation $\mathrm{R}$ is defined as $U \times V$ with membership function $\mu_{R}(x, y)=r(x, y) \cdot e^{i w(x, y)}$. Then the complement of $\mu_{R}(x, y)$ is given by

$$
C\left(\mu_{R}(x, y)\right)=[1-r(x, y) \cdot \cos w(x, y)]+i[1-r(x, y) \cdot \sin w(x, y)]
$$

And it should satisfy the periodic function defined in (16).

\subsection{Definition (Projection of a Complex Fuzzy Relation)}

Consider complex fuzzy relation $\mathrm{R}$ defined on $U \times V$

where $R=\left\{(x, y), \mu_{R}(x, y)\right\} . \forall x \in U, \forall x \in V$

i. The $1^{\text {st }}$ projection of $\mathrm{R}$ is defined as

$$
\begin{aligned}
R^{(1)} & =\left\{\left(x, \mu_{R}^{(1)}(x, y)\right)\right\}=\left\{\left(x, \max _{y} \mu_{R}(x, y)\right) \mid(x, y) \in U \times V\right\} \\
& =\left(x, \max _{y} r(x, y) \cdot e^{i \max _{y} \mu(x, y)}\right)
\end{aligned}
$$

ii. The $2^{\text {nd }}$ project is defined as

$$
R^{(2)}=\left\{x, \mu_{R}^{(2)}(x, y)\right\}=\left\{y, \max _{x} r(x, y) e^{i \max _{x} \mu(x, y)}\right\}
$$

iii. The total projection

$$
R^{(T)}=\max _{x} \max _{y}\left\{\mu_{R}(x, y) \mid(x, y) \in(U \times V)\right\}
$$

In the above, max means maximum with respect to $\mathrm{y}$ while $\mathrm{x}$ is to be considered as fixed and $\max _{x}$ maximum with respect to $\mathrm{x}$ while $\mathrm{y}$ is to be considered fixed. The projection determines the maximal boundary of the complex fuzzy sets in a complex fuzzy relation,

Example 4.1. Consider the following complex fuzzy relation.

$$
\left(\begin{array}{cccc}
0.6 \mathrm{e}^{\mathrm{i} 1.2 \pi} & 0.6 \mathrm{e}^{\mathrm{i} 1.2 \pi} & 0.6 \mathrm{e}^{\mathrm{i} 1.2 \pi} & 0.5 \mathrm{e}^{\mathrm{i} \pi} \\
0.6 \mathrm{e}^{\mathrm{i} 1.2 \pi} & 0.8 \mathrm{e}^{\mathrm{i} 1.6 \pi} & 0.8 \mathrm{e}^{\mathrm{i} 1.6 \pi} & 0.5 \mathrm{e}^{\mathrm{i} \pi} \\
0.6 \mathrm{e}^{\mathrm{i} 1.2 \pi} & 1.0 \mathrm{e}^{\mathrm{i} 2 \pi} & 0.8 \mathrm{e}^{\mathrm{i} 1.6 \pi} & 0.5 \mathrm{e}^{\mathrm{i} \pi} \\
0.6 \mathrm{e}^{\mathrm{i} 1.2 \pi} & 0.8 \mathrm{e}^{\mathrm{i} 1.6 \pi} & 0.8 \mathrm{e}^{\mathrm{i} 1.6 \pi} & 0.5 \mathrm{e}^{\mathrm{i} \pi}
\end{array}\right)
$$


International Journal of Computer Science, Engineering and Information Technology (IJCSEIT), Vol.1, No.5, December 2011

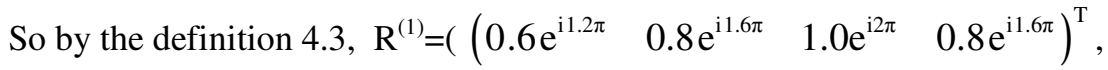

$\mathrm{R}^{(2)}=\left(\begin{array}{llll}0.6 \mathrm{e}^{\mathrm{i} 1.2 \pi} & 1.0 \mathrm{e}^{\mathrm{i} 2 \pi} & 0.8 \mathrm{e}^{\mathrm{i} 1.6 \pi} & 0.5 \mathrm{e}^{\mathrm{i} \pi}\end{array}\right)^{\mathrm{T}}$ and $\mathrm{R}^{(\mathrm{T})}=1.0 e^{i 2 \pi}$. Which shows that the maximum boundary of the given fuzzy complex relation is $1.0 e^{i 2 \pi}$. So the projection determines the maximal boundary of the complex fuzzy sets in a complex fuzzy relation,

\section{CONCLUSION}

The work presented in this paper is the novel frame work of complex fuzzy relation. In this paper the various properties and operation of complex fuzzy set as well as complex fuzzy relation are investigated. We also presented the complement of complex fuzzy relation as well as the projection of complex fuzzy relation is presented. A further study is required to implement these notions in real life applications such as knowledge representation and information retrieval in a Complex plan corresponding of two variables which is not suitable for traditional fuzzy logic.

\section{REFERENCE}

[1] Buckley J.J (1987) "Fuzzy Complex Number" in Proceedings of ISFK, Gaungzhou, China, pp.597700.

[2] Buckley J.J (1989) "Fuzzy Complex Number”, Fuzzy Sets \&Systems, vol33, pp 333-345.

[3\} Buckley J.J (1991) "Fuzzy Complex Analysis I: Differentiation”, Fuzzy Sets \&Systems, Vol 41, pp. 269-284.

[4] Jang J.S.R, Sun C.T, Mizutani E, (2005) “Neuro-Fuzzy And Soft Computing”, PHI, New Delhi.

[5] Pedrycz and Gomide F, (2004) “Introduction To Fuzzy Set Analysis And Design”, PHI, New Delhi.

[6] Ramot Daniel, Menahem Friedman, Gideon Langholz and Abraham Kandel (2003) "Complex Fuzzy Logic", IEEE Transactions on Fuzzy Systems, Vol 11, No 4.

[7] Ramot Daniel, Menahem Friedman, Gideon Langholz and Abraham Kandel, (2002) “Complex Fuzzy Set”, IEEE Transactions on Fuzzy Systems, Vol 10, No 2.

[8] Xin Fu, Qiang Shen, (2009) "A Noval Framework Of Complex Fuzzy Number And Its Application To Computational Modeling”, FUZZ -IEEE 2009, Korea, August 20-24.

[9] Zadeh L.A. (1965) "Fuzzy Sets", Information \& Control, Vol 8,pp 338-353.

[10] Zhang Guangquan, Tharam Singh Dillon, Kai-yung Cai Jun Ma and Jie Lu,(2010) “ $\sigma$-Equality of Complex Fuzzy Relations", $24^{\text {th }}$ IEEE International Conference on Advance Information Networking and Application. 
Authors

Dr. S. K. Das is working as a Reader (Associate Professor) and Head of the Department of Computer Science, Berhampur University, Berhampur, Orissa, India. He has more than 17 research publications in National and International Journals and conferences. He has supervised three Ph.D. scholars and more than 10 scholars are under supervision. He is a life member of IEEE, ISTE, SGAT, CSI and OITS. He has the research interests in the fields of Data Communication and Computer Networks, Computer Security, Internet and Web Technologies, Database Management Systems, Mobile Ad Hoc Networking and Applications.

Dr. D. C. Panda is working as an Assistant Professor in the Department of Electronics and Communication Engineering, Jagannath Institute of Technology and Management (JITM), Paralakhemundi, Orissa, India. He has more than 33 research publications in National and International Journals and conferences. He attended Several QIP of different Universities and Institutions of India. He is a reviewer of IEEE Antenna and Wave Propagation Letters and Magazine. He published a book "Electromagnetic Field Theory Simplified:- A New Approach for Beginners", which is under review. He is an exposure in Computational Electromagnetic Tools. He has the research interests in the fields of Soft Computing Techniques, Computational Electromagnetic, Microwave Engineering and MIMO Wireless Networks.

Mr. Nilambar Sethi is working as an Associate Professor in the Department of Information Technology, Gandhi Institute of Engineering \& Technology (GIET), Gunupur, Orissa, India. He is under the supervision of Dr. S.K. Das and Dr. D. C. Panda for his Doctoral degree in the field of Complex fuzzy sets and its applications. $\mathrm{He}$ is a life member of ISTE. He has the research interests in the fields of Computer Security, Algorithm Analysis and Fuzzy Logic.
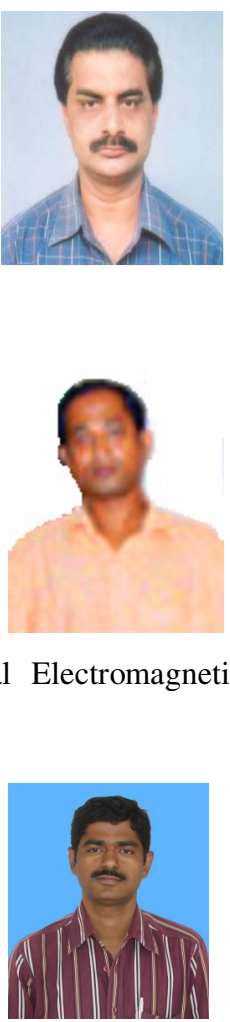

Dr. S. S. Gantayat is working as a Professor in the Department of Information Technology, Gandhi Institute of Engineering \& Technology (GIET), Gunupur, Orissa, India. He has more than 8 research publications in National and International Journals and conferences. He is guiding one student for his doctoral degree in the field of expert systems. He attended several workshops of different Universities and Institutions of India. He is a reviewer of WSES and Information Sciences. He is member and life member of IE(I), ISTE, OITS, IARCS, IACSIT, ISIAM, IAMT, IMS and SSI. He has the research interests in the fields of Fuzzy Sets and Systems,

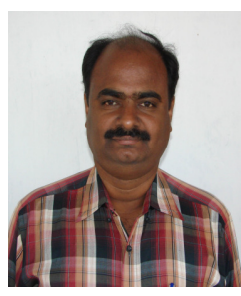

Rough Set Theory and Knowledge Discovery, List Theory and Applications, Soft Computing, Cryptography and Discrete Mathematics. 